\title{
Breast Cancer Detection Using Deep Belief Network by Applying Feature Extraction on Various Classifiers
}

\section{Prasath Alias Surendhar $\mathbf{S}^{*}$, R.Vasuki ${ }^{2}$}

${ }^{1 *}$ Research Scholar, Dept. of Biomedical Engineering, Bharath Institute of Higher Education and Research, Chennai.

${ }^{2}$ Professor \& Head, Dept. of Biomedical Engineering, Bharath Institute of Higher Education and Research, Chennai.

1*Email: mail.surendhar@gmail.com, ${ }^{2}$ Email: rkaran02@gmail.com

Article History: Received: 11 January 2021; Accepted: 27 February 2021; Published online: 5 April 2021

ABSTRACT: Among various types of cancer, breast cancer is considered as second largest hazardous diseases that cause death. Initially, small lump like structure grow from breast cells which are considered as malignant lumps. To find the available of malignancy in breast area, several checkups such as self-test and periodic has to be done to reduce the death rate due to breast cancer. But, the classification of breast cancer by medical physicians using available techniques is not sufficient, so it is important to improve the classification technique using neural network. The four important phases namely preprocessing, segmentation, feature extraction, and classification can be done in constructed Deep Belief Network (DBN) whereas preprocessing makes to remove the noise and artifacts of mammogram image and then the glands are enhanced. The preprocessed output is given to fuzzy c-means segmentation process with the help of masking. Again the feature extractors such as Scale Invariant Feature Transform (SIFT) and Speeded up Robust Transform (SURF) made to apply on two types of classifiers such as gradient boosting tree classifier and adaboost classifier. The examination is done interms of parameters such as accuracy, precision, recall and F1 score.

Keywords -Deep learning, classification, preprocessing, segmentation, feature extraction, breast cancer.

\section{INTRODUCTION}

Breast cancer is a type of malignant progress that initialize from tissues in breast which are present under the interior layer along with breast lobules or milk ducts [1]. This cancer is considered as the second widespread disease in the world, so it is significant to analyze in starting stage which tends to give proper medication for patients. Over the past decade, research in finding breast cancer is tremendously increasing [2]. Usually, the doctors use non-invasive devices along with medical imaging to find the availability of cancer in breast. While the malignancy begins, there is the possibility of being error location of flesh strains or liquid till the confirmation of malignancy [3]. The biopsy is done by inserting an operative incision or needle which may increase the level of malignancy [4]. Basically, the mammographic breast figure is typically noise remove to eliminate the unwanted areas while finding the cancer in breast. The exploration of irregularities could be consequently, be limited to the breast area by abolishes the pectoral might and conditions areas commencing the mammogram.

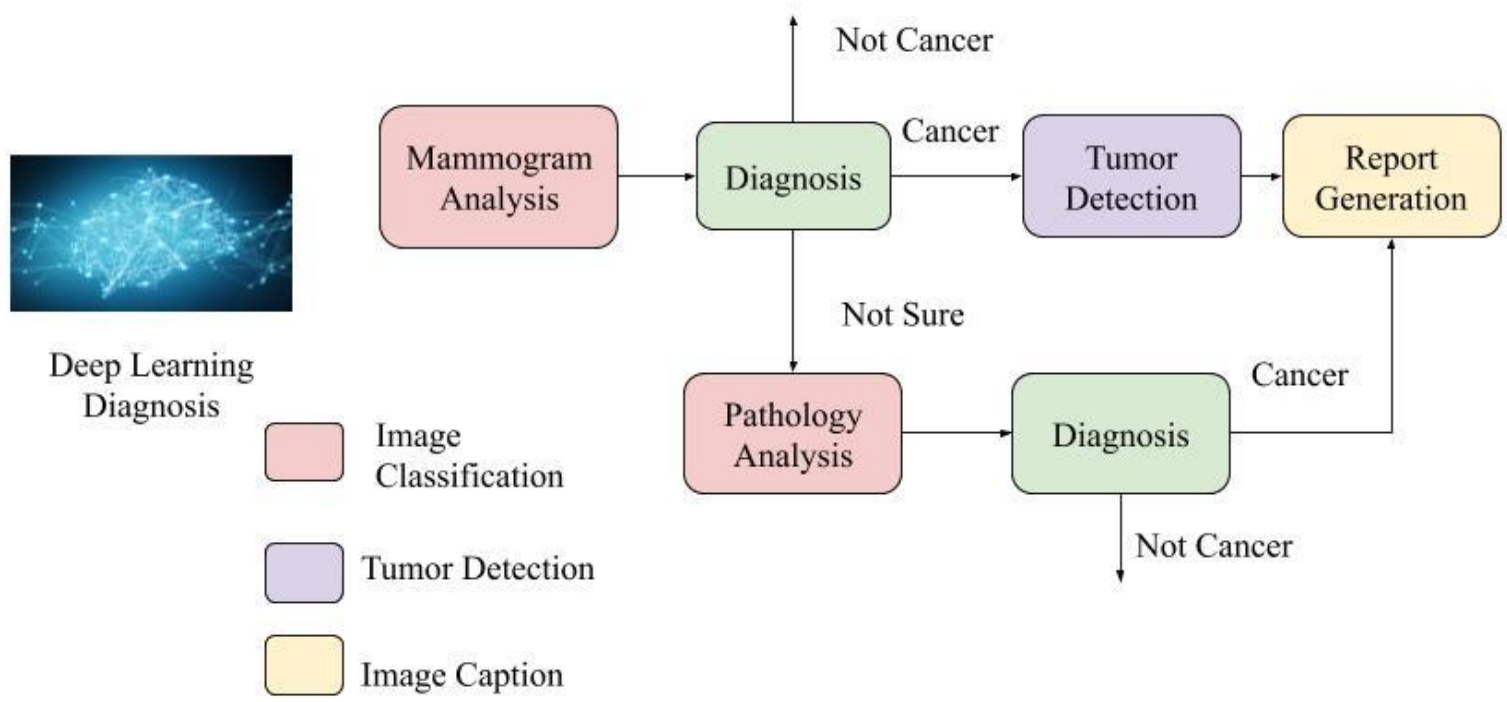

Figure1: Deep learning based breast cancer diagnosis 
The area called deep learning is comes under machine learning, which helps to enhance the medical imaging application by improving the accuracy rate of cancer detecting techniques which in clearly indicated in figure-1 [5]. There are several challenge in mammography image classification, because the occupancy of tiny portion of tumor inbreast area. Full-Field Digital Mammography (FFDM) image is usually in the choice of $6000 \times 8000$ pixels with the cancer ROI as the range of $100 \times 100$ pixels. Pre-training is a capable technique to concentrate on the difficulty of order a classifier when the perfect outsized and absolute working out datasets are not existing. In the case of Belief Network (DBN) with 3-tie architecture, pre-training has to be done to initialize the weighted input with the help of fine tuning [7]. As a result, the training accuracy and speed has been extended by the way of 3-tier architecture. This developed network needs ImageNet32 database with fine tuned model for reputation process. Even though the exact chore is not associated to the original preparation dataset, the model's power restriction are previously modified to distinguish primal skin texture, such as limits, curve and touch, that can be gladly used for a dissimilar task. This frequently keeps training period and progress the simulation's concert [8].

The motivation of this work is as follows, despite the fact that sonography is critical for finding of disease in increasing among ladies, there is right now no exploration paper with respect to the characterization of bosom malignancy that utilizes effective and vigorous CNNs. These days, the principle challenge in identifying bosom disease which is viewed as one of the most touchy techniques for imaging in atomic medication, respects assembling a calculation that naturally recognizes if a patient is experiencing threat. The calculation must be very exact since individuals' lives could be in question. Artificial Intelligence (AI) draws near, with an emphasis on profound learning calculations, have especially demonstrated a promising pertinence in clinical picture investigation in the zone of atomic medication. Notwithstanding, order precision of bosom disease, examined by AI draws near, including CNN frameworks, has not been settled at this point.

The organization of paper is as go behind, section 1 describes the overview of breast cancer, and application of deep learning in cancer detection, section 2 describes the existing techniques for breast cancer prediction with its limitation. In section 3 proposed methodology is explained with subsections like preprocessing, segmentation, feature extraction and classification are discussed with appropriate algorithm. Section 4 gives detailed experimental analysis and then section 5 ends up with conclusion and future work.

\section{LITERATURE SURVEY}

Sebai et al.,(2020) suggested a new Partially Supervised Framework (PSF)by using the combination of two deep fully convolution networks whereas, one among them is designed for practice with weak labels and the another one is based on typical weight calculative transformation. In the detection stage of designed architecture, the combination of segmentation is done with the output of mitosis consideration. The limitation is, the accuracy level is less because the proposed framework did not concentrate on optimized weight transfer function [9].

Zheng et al.,(2020) introduced Deep Learning Assisted Efficient AdaBoost Algorithm (DLA-EABA) to find availability of cancer in breast by deriving the mathematical formula or concern parameters with the sophisticated computational techniques. Moreover, this algorithm is compared with conventional classification methods with the activation of Deep Convolution Neural Networks (CNNs). This deep learning framework had numerous convolution layers, Max-pooling level. The errors while classification and evaluation is processed in softmax layer and hence the performance of this algorithm data do not cohorts have extremely connected production metrics in stipulations of the tough and preparation data sets [10].

Suresh et al.,(2019) suggested an architecture with the consideration of four significant steps like, imagepreprocessing, segmentation, feature extraction, and classification. The architecture has Laplacian filter over it which has the identification of edges in image with improved noise level. After the filtration part, the segmentation is done by Adaptively Regularized Kernel- based Fuzzy- C- Means (ARKFCM). It was a flexible great level machine learning technique to localize the object in complex template. The hybrid feature extraction was carry out on the segmented cancer region to remove feature subsets. The limitation is less classification rate, due to unused descriptor level features are multi-objective classifier [11].

Juan et al.,(2019) suggested 3D deep Convolutional Neural Network (CNN) for identify the presence of breast cancer and confine the injury at Dynamic Contrast Enhanced (DCE) data in well organized mode. The dataset such as 3D DenseNet19 was exploit as the roads of deep learning model with the consideration of trival layers. Particularly, 3D DenseNethad consists of three layers with original stalk arrangement, four thickly linked block, three conversion layers, and lastly a categorization coating for computation. In the thickly associated obstruct, all the appearance of the previous level were result as input to the last cover. The limitation is, the system taught for identifying exact abnormality since the feebly administer localization chore could only identify the lesion with a elevated growth likelihood [12].

Saha et al.,(2018) commence a deep neural network to resolve the concern of convolutional and deconvolutional fraction of the projected outline with primarily of manifold convolution deposits, max-pooling level, 
spatial pyramid pooling deposits, deconvolution layers, up-sampling layers and Trapezoidal Long Short-Term Memory (TLSTM). A automatically linked and a softmax layer were responsible for categorization and inaccuracy opinion. Lastly, the HER2 attain better classification mode. The technique extremely frequently lead to lesser correctness since it did not judge a occupied deep learning model [13].

Carneiro et al.,(2017) described an automatic method with examination of unregistered Cranio-Caudal (CC) and MedioLateral Oblique (MLO) mammography sight in organize to guess the patient's hazard of rising breast cancer. The fundamental advancement behind this philosophy lies in the utilization of profound learn replica for the issue of mutually grouping unregistered mammogram sees and particular division guides of bosom sores (i.e., ample and mini calcifications). This is a comprehensive procedure that can arrange an entire mammographic test, hold the $\mathrm{CC}$ and MLO sees and the division maps, rather than the characterization of individual sores, which is the predominant methodology in the arena. The disadvantage is, the joint examination of unregistered multi-mode (CC and MLO) and multimodal input (pictures and division maps) needed elevated highlights [14].

Yap et al.,(2016) designed deep learning methods for breast ultrasound lesion recognition with examination of three special process: a Patch-oriented LeNet, a U-Net, and a reassign learning approach with a pretrained FCNAlexNet. The Transfer Learning FCN-AlexNet attain the most excellent fallout for Dataset A and the suggested Patchbased LeNet attained the best consequences for Dataset B in conditions of F-measure. It is significant to rising the accuracy by calculation additional training facts [15].

\section{PROPOSED METHODOLOGY}

In this section, deep learning method is used to classify the cancer cells in breast images whereas, it consist of four phases namely preprocessing, segmentation, feature extraction and classification. The phases involved in Deep Belief Networks is indicated in figure 2.Here, the input images are taken form medical database are feed to preprocessing step to remove the noise and artifacts of mammogram image and then the glands are enhanced. The preprocessed output is given to fuzzy c-means segmentation process with the help of masking. The spatial convolution process is involve hereto examine the linear combination of a series of discrete 2-dimensional data which is then performed by frequency domain. The segmentation is done and hence the output is given for feature extraction, which consists of two methods namely Scale Invariant Feature Transform (SIFT) and Speeded Up Robust Transform (SURF). The feature extracted output is given to gradient boosting tree classifier and adaboost classifier.

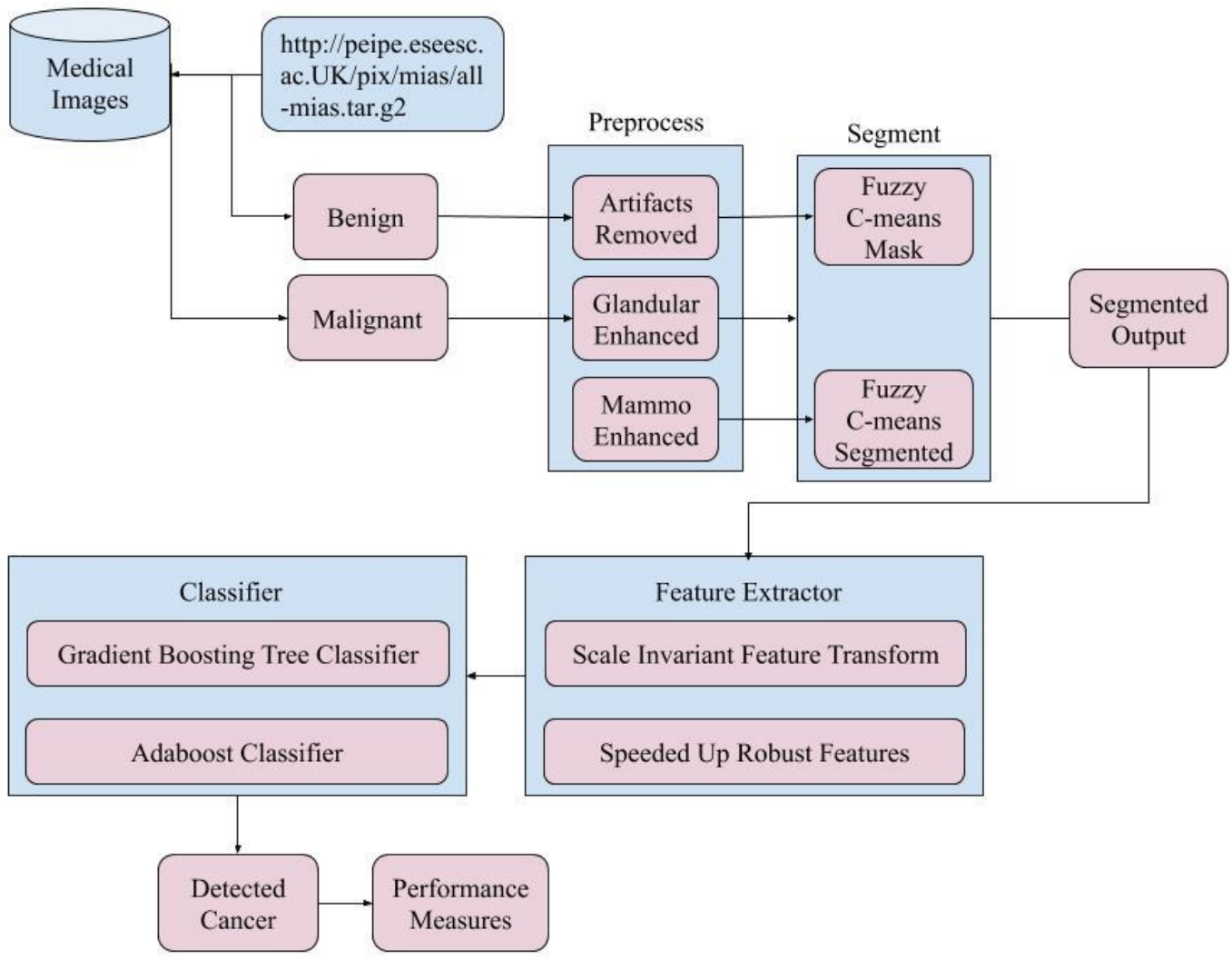

Figure 2:Steps involved in Deep Belief Networks 


\subsection{PREPROCESSING}

Preprocessing helps to enhance the quality of mammogram image by eliminate the noisy and unwanted parts in background. This enhancement helps to minimize the complication while interpreting. This pre-processed image is input for segmentation and feature extraction step.

\subsubsection{Artifacts removal}

Basically, the image acquisition is significant for analyzing grey scale images for the purpose of noise and artifact reduction. The artifacts which is also a type of noise, that can be remove by efficient filtering technique such as 2D median filter. Once the filtration is done, the grey level images are converted in to binary images with the appearance of white and black mask. The aperture procedure hence reduce the abnormal areas in breast whereas, the removal of artifacts are base on calculating the threshold range of each image.

\subsubsection{Glandular enhancement}

The attenuation level of mammogram images are less due to increased tissue levels, specifically the tissue level for adolescent women are more. This makes confusion among radiologists to detect cancer by efficiently extract the feature of breast with improving the accuracy rate. Nevertheless, the overall categorization of many tissue remnants an unaddressed chore; and it turn out to be additional hard for the radiologist to distinguish among usual and cancerous tissues in term of small size malignancies.

\subsubsection{Mammo enhancement}

The process of mammogram enhancement is the process of improving the contrast of images by reducing the noise to detect the abnormalities. One of the contrast improvement technique named as statistical based pixel intensity with confined neighborhood are fixed to initialize the property such as size, color with respect to multiscale processing. The process $f$ enhancement for a image is indicated in figure- 3 with the consideration of four important steps:

- Neglect the image's background and extent its resolution representation.

- Introduce the matrix product of each resolution.

- Now, each resolution is deliberated with matix blocks whereas the nolinear function is coincide with equivalent constrains value. As a result, the enhanced image is produced.

- Rescale the produced image and combine them to form the resultant part.

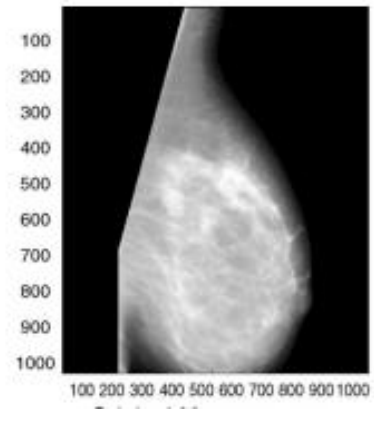

Figure 3(a)

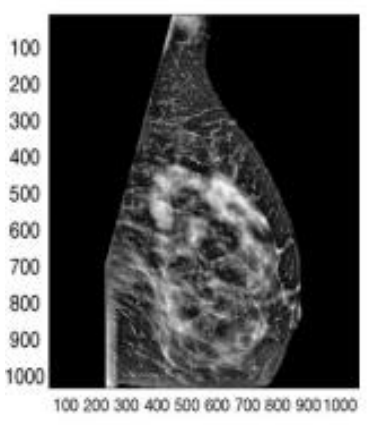

Figure 3(b)

\subsection{SEGMENTATION}

Figure 3: (a) and (b) Mammogram enhancement

Basically, mammo image segmentation is the process of dividing reciprocally uniformed area by initializing the Region of Interest (RoI).

\subsubsection{Masking}

Under the Deep Belief Networks (DBN), the masking is prepared by Region Proposal Network (RPN) with RoIPool for the purpose of removing the features with the extension of boundary boxes. The pooling method with various scaling sizes are also quantified for each layer with Region of Interest (RoI).By doing so, the limitation of losing spatial information is reduced. Initially, the training images are categorized to compose the testing and validation sets, afterwards a new mode is recognized by ensuring the accuracy and stability constant. Finally, the Loss Function (LF) for masking in DBN is given as $L F=L_{\text {class }}+L_{\text {box }}+L_{\text {mask}}$. This constructed masking's loss function is regularized for all the network model. The trained model was functional to calculate and investigate with new data with confirmation process.

The loss function of masking in DBN is distinct as follows: 


\subsubsection{Fuzzy c-means segmentation}

$$
\mathrm{L}=L_{\text {class }}+L_{\text {box }}+L_{\text {mask }}
$$

This segmentation is done by partition matrix by fixing the values between 0 and 1 with the representation of membership's degree for every image with clustering centers. Finally, the objective function is given as,

Where, $m$ is any real number greater than one.

$$
\operatorname{Jm}(\mathrm{M}, \mathrm{N})=\sum_{i=1}^{c} \sum_{k=1}^{n} \frac{u i k(m)}{n} x k-c i
$$

$\mathrm{X} 1, \mathrm{X} 2, \mathrm{Xn}$ are $\mathrm{n}$ data sample vectors.

$\mathrm{N}=\{\mathrm{N} 1, \mathrm{~N} 2, \ldots \mathrm{Nc}\}$ are cluster center. $\mathrm{M}=$ uik is a $\mathrm{m} \times \mathrm{n}$ matrix, where uik is the ith membership value of kth input sample Xk such that

$$
\sum_{k=1}^{n} \frac{u i k(m)}{n}=1
$$

The feature for exponential weight maximizes the overall fuzziness of the membership function representation of |.|, which makes the similarities between input image with its corresponding cluster center. By finding the optimization value for objective function membership uik, and cluster center $\mathrm{Ci}$ can be updated as

$$
\begin{aligned}
& \text { uik }=\frac{1}{\sum_{j=1}^{c} \mid \frac{|\mathrm{kk}-\mathrm{ci}|}{|\mathrm{ck}-\mathrm{c}|}} \\
& \mathrm{ci}=\frac{\sum_{k=1}^{n} u i k(m) \cdot x k}{\sum_{k=1}^{n} u i k(m)}
\end{aligned}
$$

\subsection{FEARURE EXTRACTION}

For efficient mammogram image classification, the feature extraction is significant. In the traditional classification method, the feature of the images are compounded based on some specific rules with respect to texture, detector, and statistical level. Texture features essentially stand for the low-level feature information of an image, which offer more comprehensive sequence of an image that may be potential from histogram information alone.

\subsubsection{Scale Invariant Feature Transform (SIFT)}

After all pixel values from an segmented mammogram are converted in double data type, then the image histogram is analyzed. The mentioned histogram specifications allow in order for obtaining an image with lower dynamic gray-level range and an image with higher dynamic gray-level range from the original mammogram image. SIFT keypoints are extracted on both two versions of the image considering different aspects that will be described below. We deliberately discard the keypoints having negative Laplacian values because of points located close to the edge of the breast.

$$
\begin{aligned}
& \mathrm{F}(\mathrm{x} / \mu, \alpha)=\frac{\exp \left\{\frac{x-\mu}{\alpha}\right\}}{\alpha\left(1+\exp \left\{\frac{x-\mu}{\sigma}\right\}\right.} \\
& \mathrm{F}(\mathrm{x}, \mathrm{h})=\frac{1}{n h} \sum_{i=1}^{n} k\left\{\frac{x-x i}{h}\right\}
\end{aligned}
$$

The extraction of SIFT is mainly characterized by two parameters: the peak threshold and the edge threshold. The edge threshold allows eliminating peaks of the Difference of Gaussians (DoG) scale space with small curvature. The peak threshold parameter filters out the peaks of the DoG space scale, showing low absolute values. The output of the SIFT-based technique is a set of SIFT key points that identify the candidate suspicious regions.

\subsubsection{Speeded Up Robust Transform (SURF)}

This method consists of interest point based local image pixels with the consideration of SURF descriptor. This descriptor is based on hessian -oriented blob sensors are utilized to discover the point of interest beginning at every mammographic region of interest images. Initially, the perceived points of interest are splitted in the range of $(4 \times 4)$ metre square, and hence the extraction process is done by using Haar wavelet method which is improved to the $(5 \times$ 5) range. For enhance this technique, method named as invariance method is applied with the orientation of higher determinant level of matrix at both $\mathrm{x}$ and y directions. The radius is unchanging i.e., $6 S$ where $S$ signify the measure of point of interest. The response are biased with a Gaussian of $\sigma=3$.2. Hence all the standards are practically resolute.

\subsection{CLASSIFICATION}

The inputs for supervised and un-supervised deep learning systems based classification are Deep Invariant Features (DIFs). This makes to use in Deep-Learning Neural Network (DL-NN) model with distribution oriented supervised and un-supervised layers. DL-NN is depend upon hierarchical basis with a greedy layer-by-layer 
approach abstraction level is constructed for knowledge. The DL-NN has many application in carry out such as linguistic demonstrating and mainframe image, picture cataloging and speech acknowledgment.

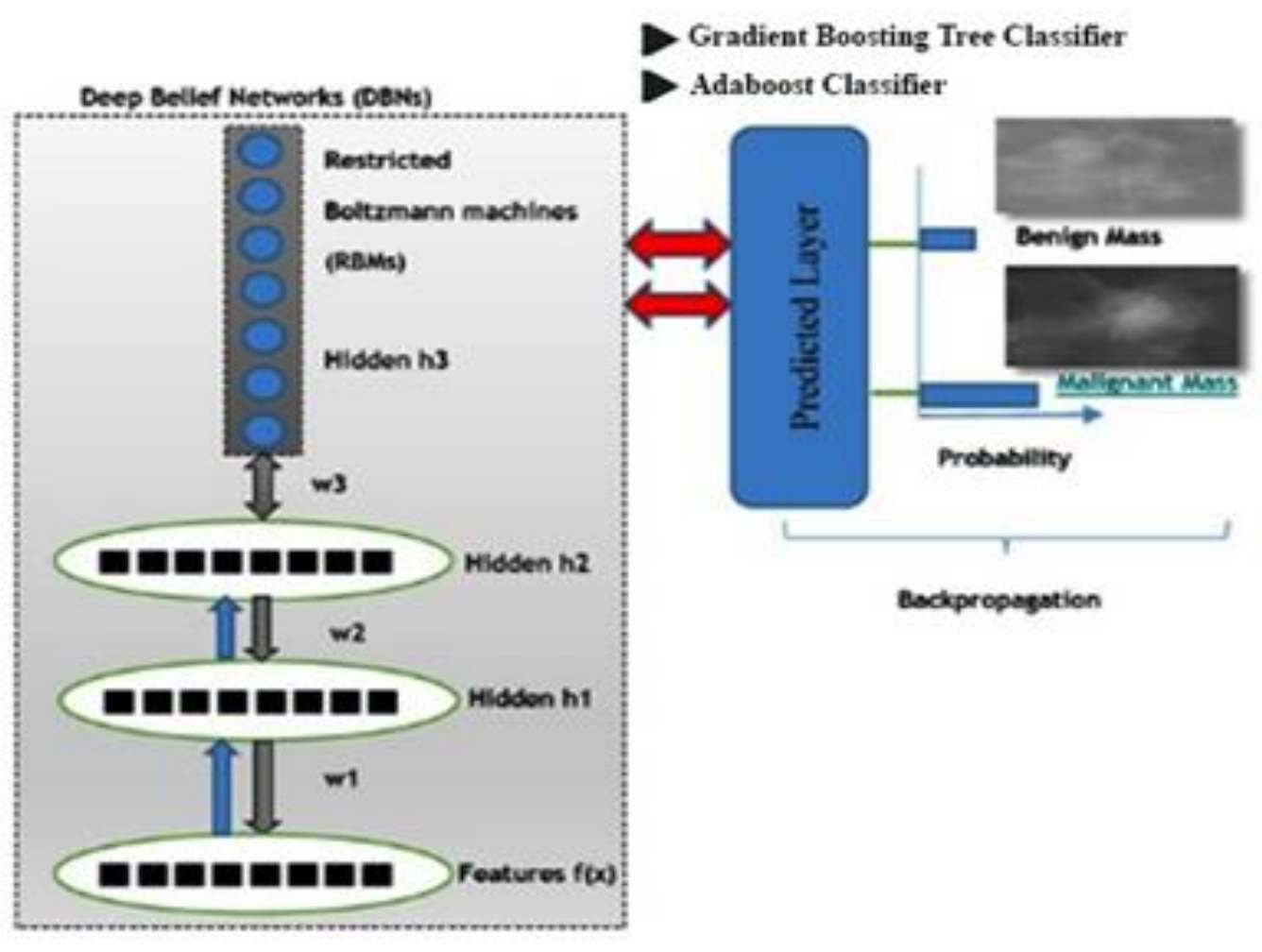

Figure 4: Classification in deep belief networks

Figure 4 shows the application of DL-NN algorithms for classification purpose with the consideration of optimization step in three layers. At particular time, only one layer is design to learn and hence after the completion of it, we can go for another layer and so on. The overall performance of classification is improved by including the fine tuning step on the entire network with the suggestion of back broadcast fault deviation model. The final classification decision is conducted by softmax linear regression model.

The numerical demonstration of DBN to classical types and $\mathrm{n}$ hidden layers $\mathrm{hn}$ can be defined as:

$$
\mathrm{P}(\text { features, hn })=(\text { hi } \mid \text { hi }+1) \times \mathrm{P}(\mathrm{hn}-2, \mathrm{hn})
$$

The training processing the network is initiated by means of an unsupervised greedy layer-wise model along with invariant features. The initial layer is opted for input features. The secod layer named as unsupervised visible layer is performed with mean activation function of the training samples. Finally the entire output is iterated with preferred range of propagation in upward direction activation function.

\subsubsection{Gradient boosting tree classifier}

Consider the set of training images which is represent as ' $y$ ' for ' $n$ ' number of input breast images. These training datasets are working based on extracted features that combines ensemble classifiers for making the classification to be strong enough by neglecting the false positive rate. After this, all the comparable weights are computed for base classifier with training loss by finding the difference between actual and observed value. Based on the error value, the first weights of all the classifiers are reduce or improved. lastly, the steepest descent function discover the most outstanding classifier with smallest amount training loss among the more than a few base classifiers. This procedure add to the precise categorization and wrong categorization

\subsubsection{Adaboost classifier}

The role of AdaBoost algorithm along with classifier helps to improve the accuracy by enhancing the binary classification with the division of positive and negative basis. Let's regard as the rest of preparation example $\{(y j$ ', $x j)\} j=1 n$, where $y j$ ' specify the training samples and $x j$ is a boolean assessment allocate based on clinical data of cancer sufferers throughout the dataset preprocessing pace. AdaBoost is an capable method that perk up 
the classification correctness an by transform a weaker categorization set of $\left\{h\left(y^{\prime}\right)\right\}$ into a higher classification of $h\left(y^{\prime}\right)$. Here, the decision stump is considered as efficient learning algorithm which is hence combine with adaboost classifier. The result of $h\left(y^{\prime}\right)$ is 1 if $y^{\prime}$ is classified as a positive instance and or else 0 . This deviating restrictions fragile classifiers to support on a solitary characteristic $f i$ only which is an consequence, for each weak classifier that include a solitary characteristic , a threshold $\theta i$ and a parity $q i$ which whichever to 1 , consequently, denoting inequality path.

The adaboost method suggests the approximate positive rate for $\theta i$ and $q i$ for every feeble classifier, which is hence represented as $h\left(y^{\prime}\right)$. In order to evaluate the feeble classifier, it is significant to evaluate all the available classifiers by making the combination of $q i$ and , for which the figure is controlled only on an never-ending numeral of preparation

$$
(\mathrm{qi}, \Omega 1)=\arg \min \sum_{j=1}^{n} \mathrm{hi}\left(\mathrm{y}^{\prime}\right)-\mathrm{xj}
$$

\subsection{ALGORITHM}

Input: $\mathrm{A} 1, \mathrm{~A} 2, \mathrm{~A} 3, \mathrm{~A} 4, \ldots . \mathrm{An}$

Output: classified image for cancer detection

Step-1 Preprocessing

$$
\begin{aligned}
& A 1=(a 1 i, a 1 j), A 2=(a 2 i, a 2 j) \ldots \ldots A n=(\text { ani,anj }) \\
& \text { For each } \\
& \qquad y(m, n)=\operatorname{median} \sum_{a=1}^{n} x[i, j],(i, j) € w \\
& C<-y(m, n)
\end{aligned}
$$

Step-2 select the amount of clusters $\mathrm{C}$

Step -3 select the example weight $m$

Step-4 Resetting the membership uik

Step-5 calculate the cluster of each cluster Ci (Eq. 4)

Step-6 modernize the membership uik (Eq. 5) for $\mathrm{i}=1,2, \ldots, \mathrm{c}$ and $\mathrm{k}=1,2, \ldots, \mathrm{n}$,

Step-7 replicate steps 5, 6 until the alteration is less than a particular cost.

Step-8 Extract the features

$$
\begin{aligned}
& \mathrm{F}(\mathrm{x} / \mu, \alpha)=\frac{\exp \left\{\frac{x-\mu}{\alpha}\right\}}{\alpha\left(1+\exp \left\{\frac{x-\mu}{\sigma}\right\}\right.} \\
& \mathrm{F}(\mathrm{x}, \mathrm{h})=\frac{1}{n h} \sum_{i=1}^{n} k\left\{\frac{x-x i}{h}\right\}
\end{aligned}
$$

Step-9 Construct 2 classifier $\{\mathrm{B} 1(\mathrm{x}), \mathrm{B} 2(\mathrm{x}), \mathrm{B} 3(\mathrm{x}), \ldots . \mathrm{Bn}(\mathrm{x})\}$ with features

Step-10 Compute Kullback-Leibler divergence d [p (f)/q(f)] splits the images into different classes Nor or AbNor

Step-11 Construct Adaboost classifier

$$
\left\{\left(\boldsymbol{y} \boldsymbol{j}^{\prime}, \boldsymbol{x} \boldsymbol{j}\right)\right\} \boldsymbol{j}=\text {, where } \boldsymbol{x} \boldsymbol{j}=\mathbf{0}, \mathbf{1}
$$

For $\boldsymbol{r}=, \ldots \boldsymbol{R}$ do

standardize the weights $\boldsymbol{\omega r}$, , in amount to $\sum \boldsymbol{\omega r}, \boldsymbol{j n} \boldsymbol{j}=\mathbf{1}=\mathbf{1}$

For all feature, train a weak classifier $\boldsymbol{h i}$.

For error $\boldsymbol{\epsilon i}$ of a classifier $\boldsymbol{h i}$ is designed matching to the weight $\boldsymbol{\omega r}, \ldots, \boldsymbol{\omega r}, \boldsymbol{n}$ :

$$
\epsilon i=\sum \omega r, j\left|h i\left(y j^{\prime}\right)-x \boldsymbol{j}\right| \boldsymbol{n j}=1
$$

decide on the classifier $\boldsymbol{h i}$ with the minimum error $\boldsymbol{\epsilon i}$ and set $(, \boldsymbol{\epsilon r})=(\boldsymbol{h i}, \boldsymbol{\epsilon i})$.

Update the weights $\boldsymbol{\omega r}+\mathbf{1},=(\boldsymbol{\omega r},-\boldsymbol{e j})$, where $\boldsymbol{\alpha r}=\boldsymbol{\epsilon r} \mathbf{1}-\boldsymbol{\epsilon r}$ and $\boldsymbol{e j}=\mathbf{0}$ while instance $\boldsymbol{y} \boldsymbol{j}$ ' is properly classified by $\boldsymbol{h} \boldsymbol{r}$ and 1 ,

End for

Step-12 Combine 2 classifiers $\mathrm{y}=\sum_{i=1}^{n} \mathrm{Bi}(\mathrm{x})$

for each $\mathrm{Bi}(\mathrm{x})$

Initialize similar weight $\mathrm{v}(\mathrm{t})$

Compute Gaussian training loss $\beta$ [ y,Bi(x)]

Update the initial weights $v(i)$

If $[\mathrm{v}(\mathrm{i}-1)]$ then properly categorizes the dataset as Nor or AbNor

end if

else falsely categorizes the dataset as Nor or AbNor

Step- 13 discover classifier with lowly training loss arg $\min \beta[y(\mathrm{i}), \mathrm{Bi}(\mathrm{x})$

End for 
Step-14 Obtain strong classification results $\sum_{i=1}^{n} \operatorname{Bi}(\mathrm{x}) v(i)^{\prime}$

End for

End

\section{PERFORMANCE ANALYSIS}

The experimental result is carried out in Python software and the operational parameters used for analysis are indicated in table1. Comparison of feature extraction methods namely Scale Invariant Feature Transform (SIFT), Speed Up Robust Transform (SURF) are done with gradient boost classifier, adaboost classifier and Multi Layer Perceptron (MLP) classifier with respect to parametric metrics like accuracy, Precision, f1 score, recall are chosen.

Table 1: Operational parameters

\begin{tabular}{|c|c|}
\hline Operational Parameters & Values \\
\hline Epochs & 150 \\
\hline Batch Size & 16 \\
\hline hidden layers & 7 \\
\hline Learning Rate Drop & $1 \mathrm{e}-2$ \\
\hline Learn Rate Drop Period & 40 \\
\hline Initial Learn Rate & $1 \mathrm{e}-2$ \\
\hline Batch normalization epsilon & $10^{-4}$ \\
\hline Loss function error & $\mathrm{L}=L_{\text {class }}+L_{\text {box }}+L_{\text {mask }}$ \\
\hline
\end{tabular}

The parameters such as accuracy, precision, recall and F measure used for analysis is discusses as follows:

Accuracy indicates the general prediction capability of the projected deep learning model. True positive (TP) and true negative (TN) compute the capacity of classifier form to calculate the lack and being there of breast cancer. False positive (FP) and false negative (FN) recognize the amount of false predictions produce by the models.

$$
\text { Accuracy }=\frac{T P+T N}{T P+T N+F P+F N}
$$

Precision indicates overall achievement of the cancer classification model, correspondingly. It is the likelihood of a classification function which is forecast to outcome as true positive rate at the presence of disease. It is also recognized as true positive(TP) amount and can be compute as:

$$
\operatorname{Precision}(P)=\frac{T P}{T P+F P}
$$

Recall indicates the likelihood of a classifier that achieves outcome as negative at the absence of disease. It is otherwise named as true negative $(\mathrm{TN})$ rate, and can be calculate as:

$$
\operatorname{Recall}(R)=\frac{T P}{T P+F N}
$$

F1- Score is exploit to establish the prediction performance. It is constructed by analyzing the harmonic part of the precision and recall. A computed score value of 1 is measured as most excellent and if it is 0 , results in bad. Fmeasures wont consider the true negative rate in its account. The F1-Score can be calculated as:

$$
F 1-\text { Score }=\frac{2 * P * R}{P+R}
$$

The table 2 demonstrate the analysis of contrast of various classification technique. The classification is used to perform two different features such as SIFT and SURF correspondingly.

Table 2: Comparison of various classification methods

\begin{tabular}{|c|c|c|c|c|c|}
\hline Features & Classifier & Accuracy & Precision & Recall & F1-Score \\
\hline \multirow{2}{*}{ SIFT } & GradientBoostingClassifier & $83.93 \%$ & $88.89 \%$ & $81.63 \%$ & $82.5 \%$ \\
\cline { 2 - 6 } & AdaBoostClassifier & $88.39 \%$ & $88.16 \%$ & $88.32 \%$ & $88.23 \%$ \\
\hline \multirow{2}{*}{ SURF } & GradientBoostingClassifier & $91.96 \%$ & $92.19 \%$ & $91.5 \%$ & $91.77 \%$ \\
\cline { 2 - 6 } & AdaBoostClassifier & $95.54 \%$ & $95.57 \%$ & $95.35 \%$ & $95.45 \%$ \\
\hline
\end{tabular}




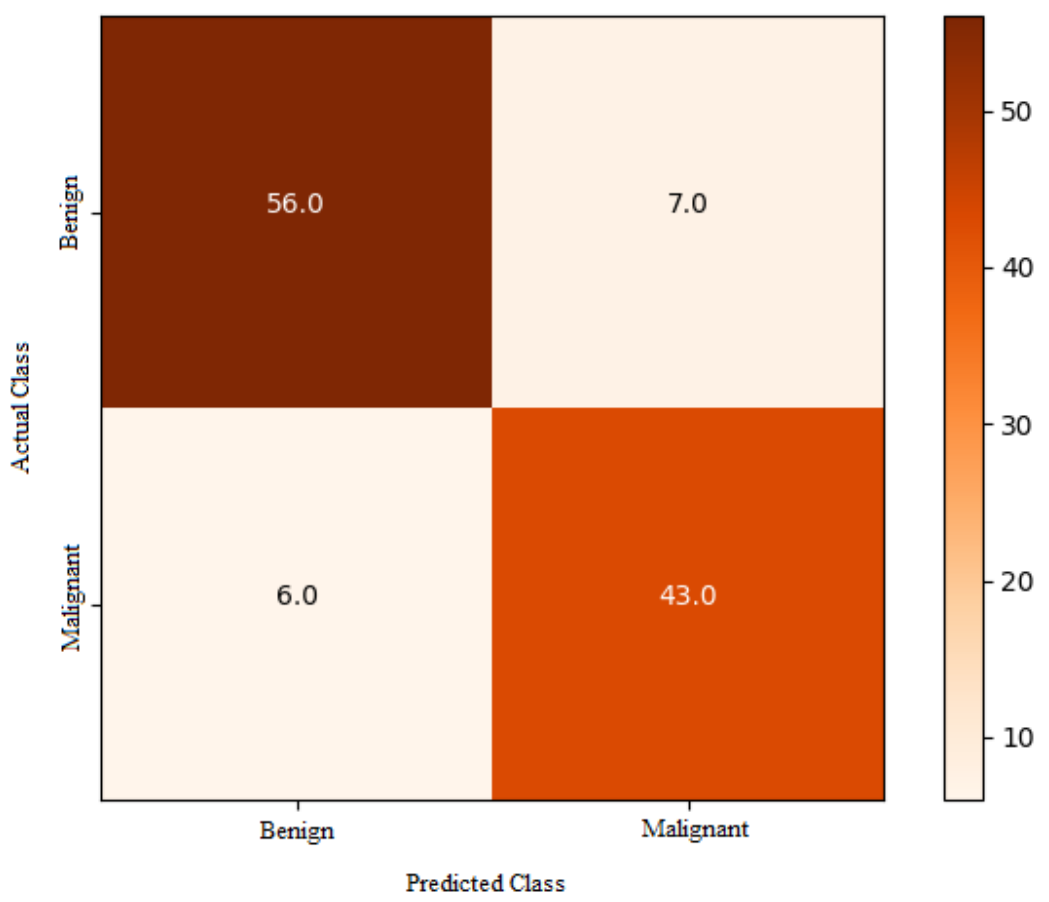

Figure 5: Confusion Matrix for SIFT feature using adaboost classifier

The above figure 5 shows the confusion matrix for SIFT features using adaboost classifierin which the rows represent the predictedclass (output class) and columns denotes the actual class (target class) of data pertaining to cancer detection. The diagonal maroon and orange that are correctly and incorrectly classified.The column on the right side indicates every predicted class while the rowat bottom represents the performance of every actual class.

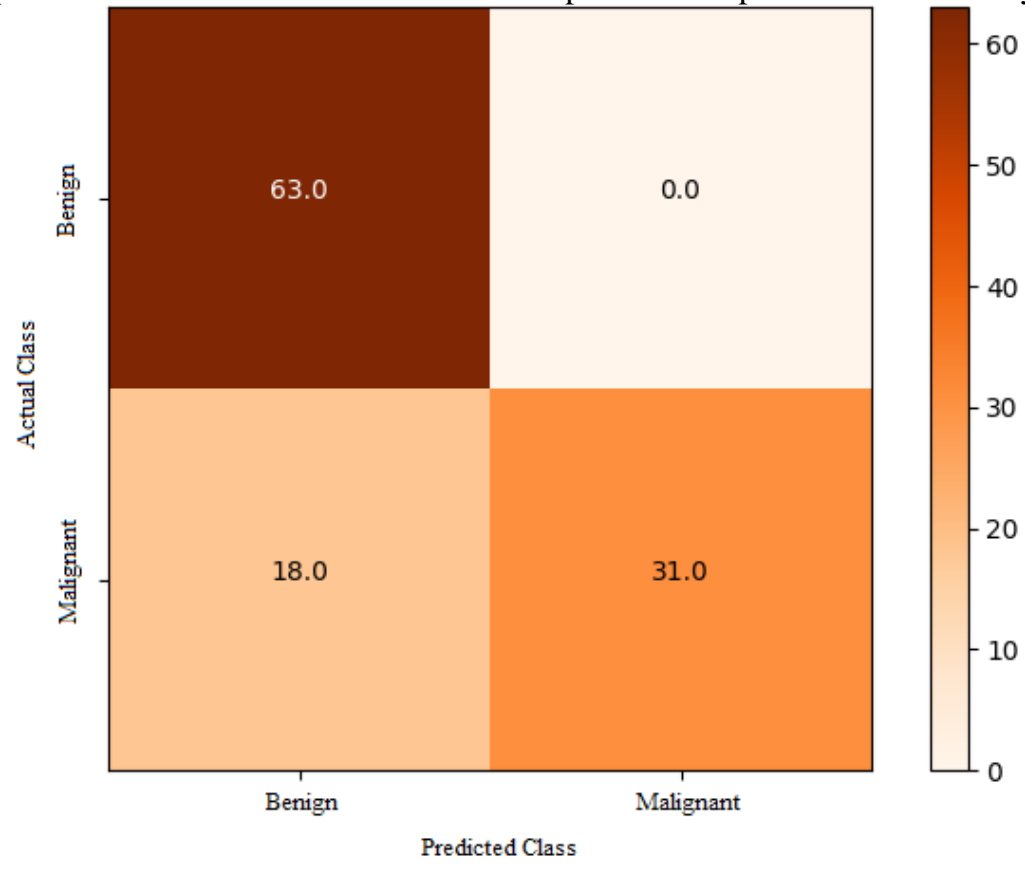

Figure 6: Confusion Matrix for SIFT feature using gradient classifier

The above figure 6 shows the confusion matrix for SIFTfeatures using gradient classifier in which the rows represent the predictedclass (output class) and columns denotes the actual class (target class) of data pertaining to cancer detection. The diagonal maroon and orange denote that, they are correctly and incorrectly classified.The column on the right side indicates every predicted class while the rowat bottom represents the performance of every actual class. 


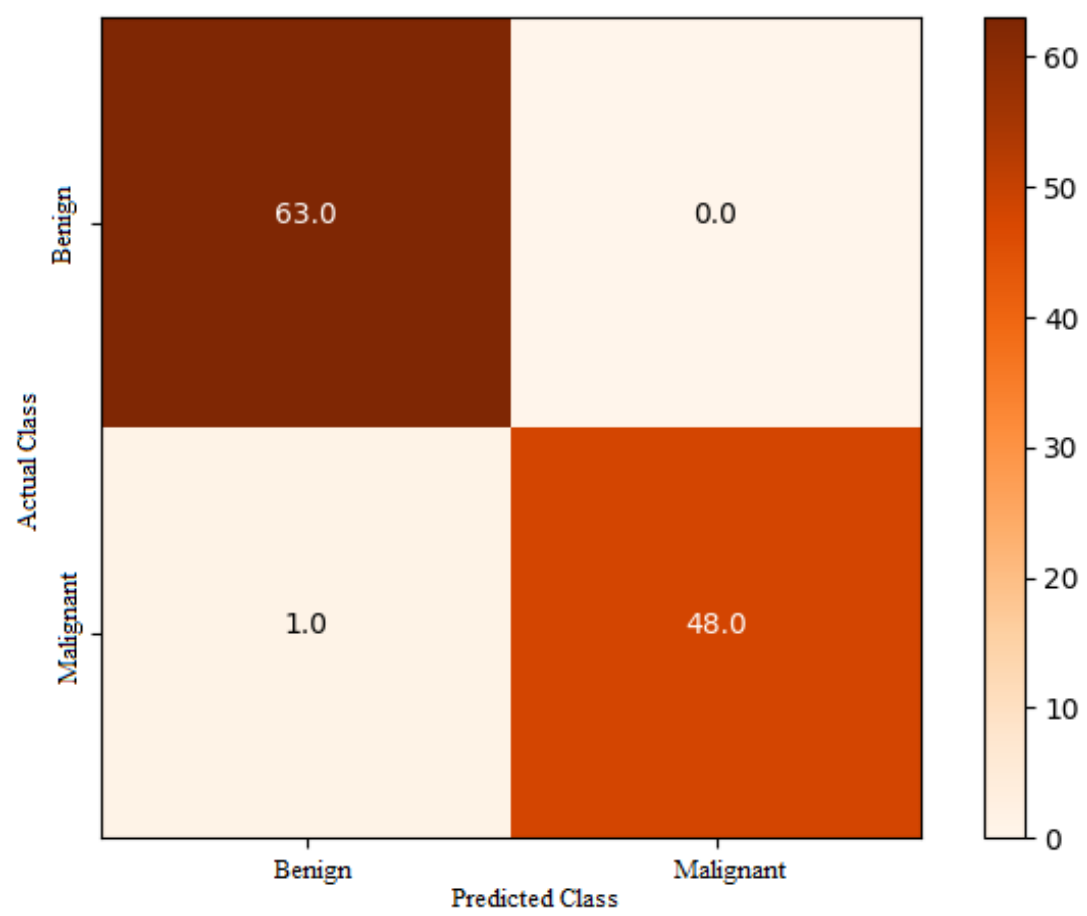

Figure 7: Confusion Matrix for SIFT feature using MLP classifier

The above figure 7 shows the confusion matrix for SIFTbfeatures by means of MLP classifier in which the rows represent the predictedclass (output class) and columns denotes the actual class (target class) of data pertaining to cancer detection. The diagonal maroon and orange denote that, they are correctly and incorrectly classified.The column on the right side indicates every predicted class while the rowat bottom represents the performance of every actual class.

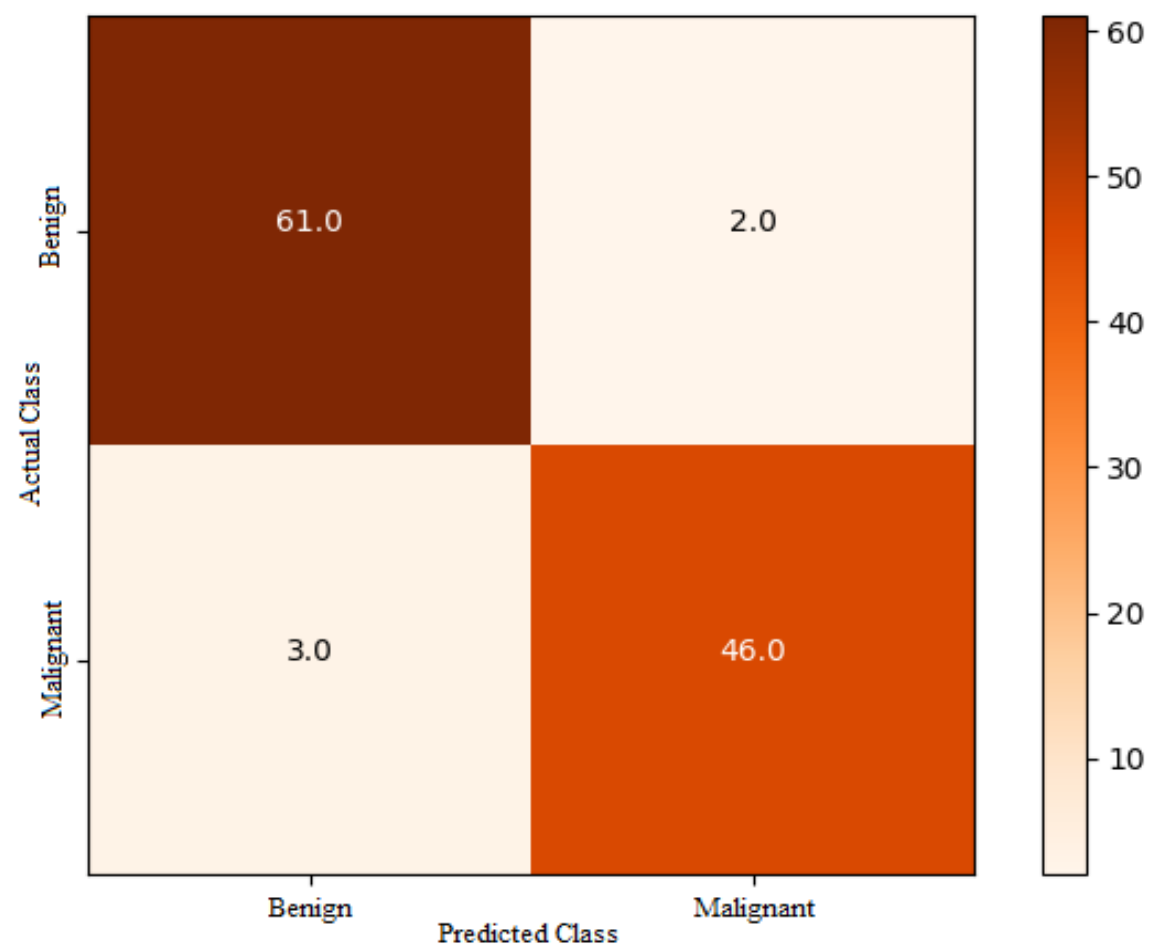

Figure 8: Confusion Matrix for SURF feature using adaboost classifier

The above figure 8 illustrate the confusion matrix for SURF feature using adaboost classifier in which the rows represent the predicted class (output class) and columns denotes the actual class (target class) of data pertaining 
to cancer detection. The diagonal maroon and orange denote that, they are correctly and incorrectly classified.The column on the right side indicates every predicted class while the rowat bottom represents the performance of every actual class.

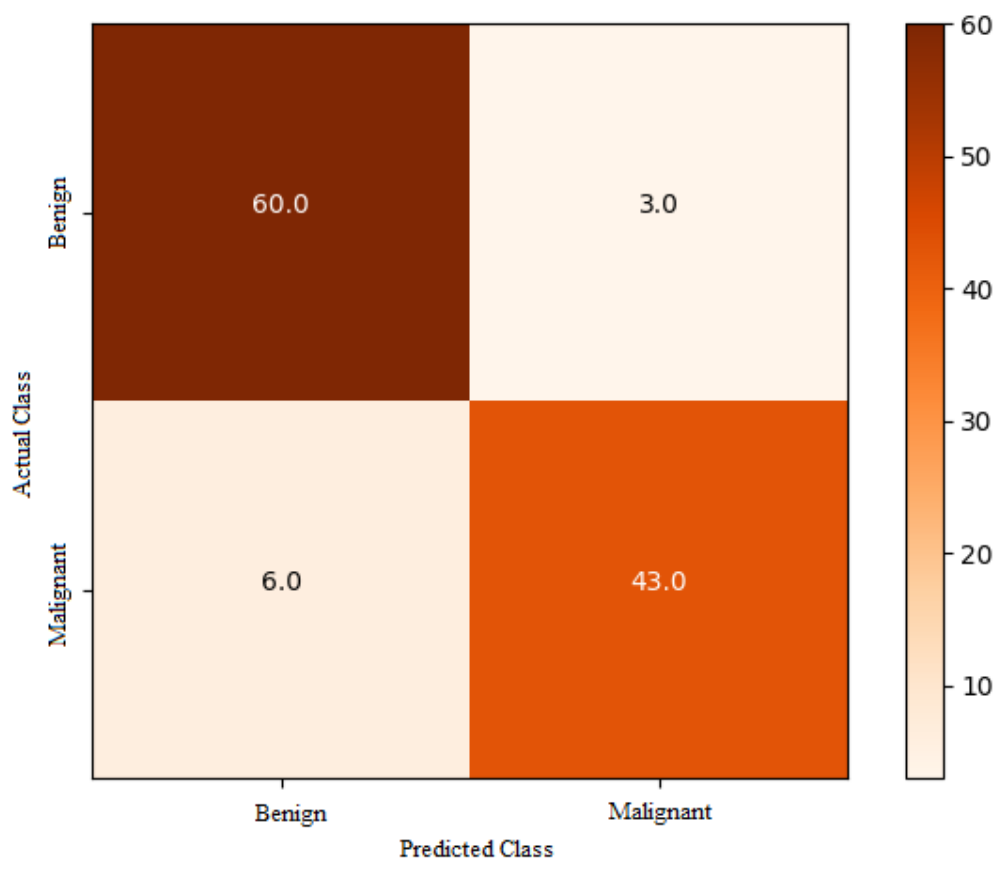

Figure 9: Confusion Matrix for SURF feature using gradient classifier

The above figure 9 demonstrate the confusion matrix for SURF feature using gradient classifier in which the rows represent the predicted class (output class) and columns denotes the actual class (target class) of data pertaining to cancer detection. The diagonal maroon and orange denote that, they are correctly and incorrectly classified. The column on the right side indicatesevery predicted class while the rowat bottom represents the performance of every actual class.

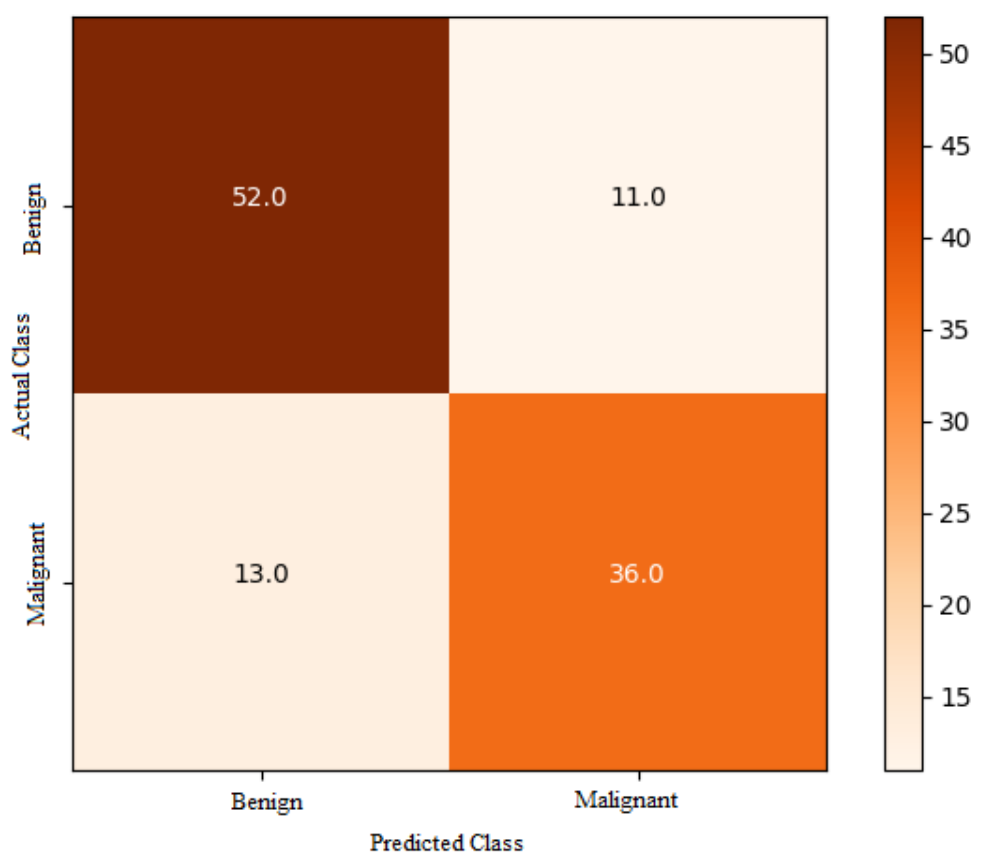

Figure 10: Confusion Matrix for SURF feature using MLP classifier 


\section{Breast Cancer Detection Using Deep Belief Network By Applying Feature Extraction On Various \\ Classifiers}

The above figure 10 shows the confusion matrix for SURF feature using MLP classifier in which the rows represent the predicted class (output class) and columns denotes the actual class (target class) of data pertaining to cancer detection. The diagonal maroon and orange denote that, they are correctly and incorrectly classified.The column on the right side indicates every predicted class while the rowat bottom represents the performance of every actual class.

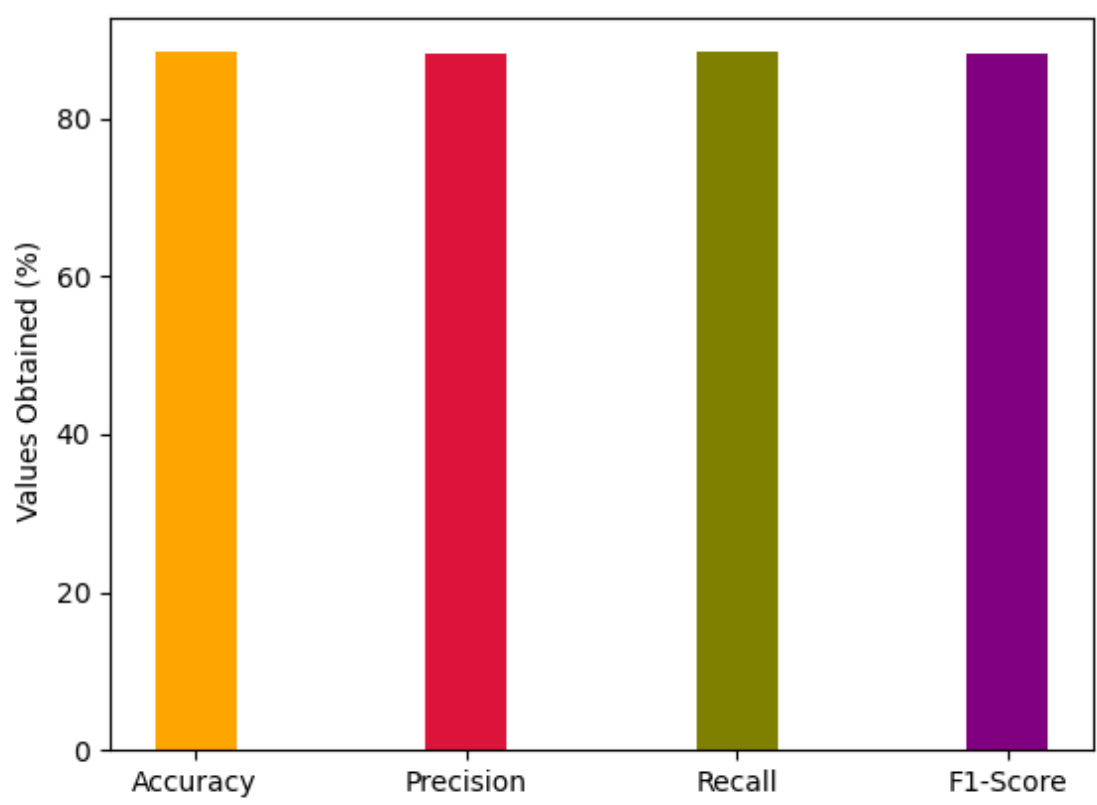

Figure10: SIFT feature using adaboost classifier

The figure 10 shows the SIFT feature using adaboost classifier whereas, $\mathrm{X}$ axis shows the Parameters to be used for analysis and $\mathrm{Y}$ axis shows the values obtained in percentage. The classifier achieves $88.39 \%$ of accuracy, $88.16 \%$ of precision, $88.32 \%$ of recall and $88.23 \%$ of F1 score.

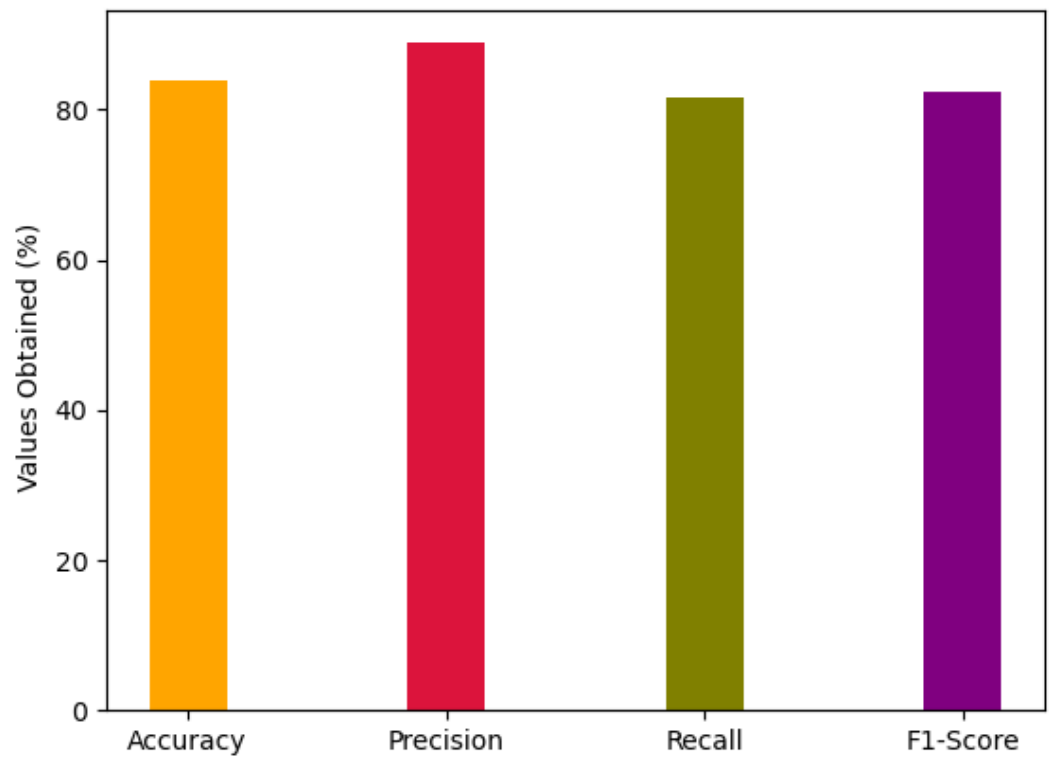

Figure 11: SIFT feature usinggradient boosting classifier

The figure 11 shows the SIFT feature usinggradient boosting classifier whereas, $\mathrm{X}$ axis shows the parameters to be used for analysis and $\mathrm{Y}$ axis shows the values obtained in percentage. The classifier achieves $83.93 \%$ of accuracy, $88.89 \%$ of precision, $81.63 \%$ of recall and $82.5 \%$ of F1 score 


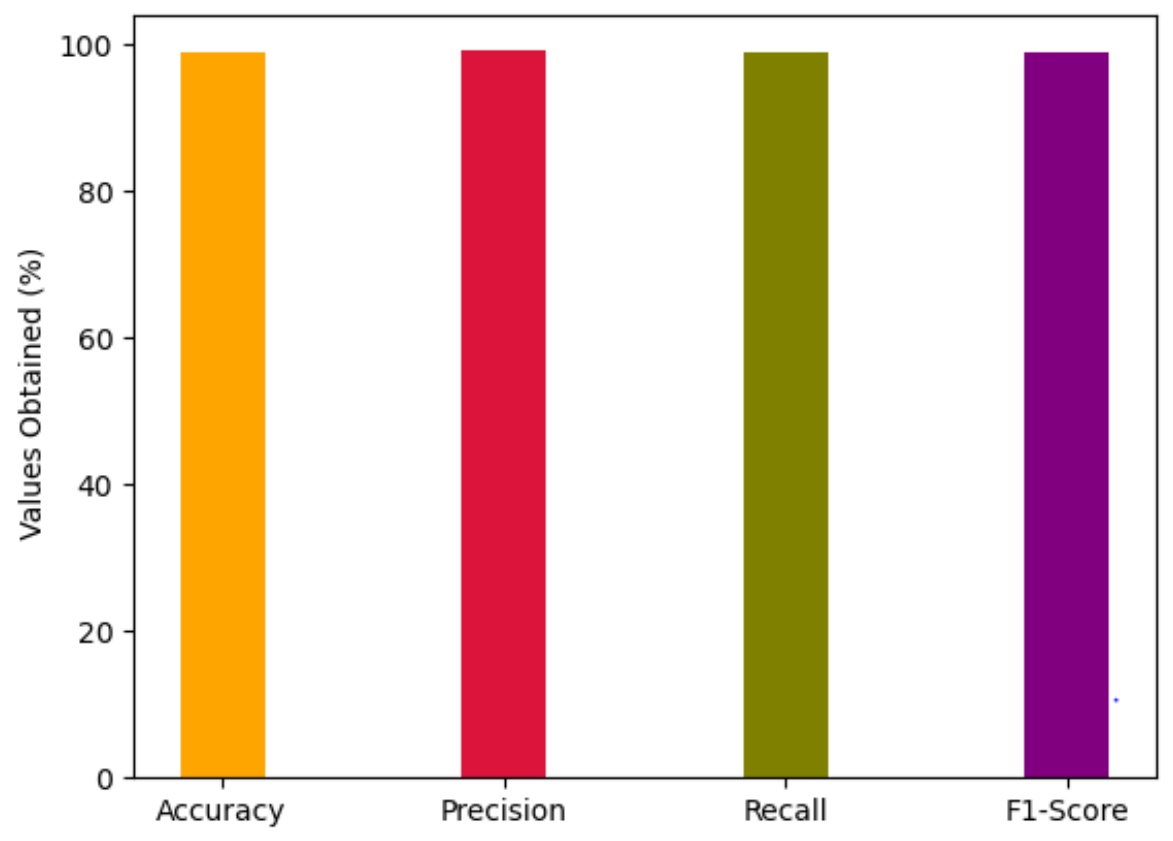

Figure 12: SIFT feature usingMLP classifier

The figure 12 shows the SIFT feature usingMLP classifier whereas, $X$ axis shows the parameters to be used for analysis and $\mathrm{Y}$ axis shows the values obtained in percentage. The classifier achieves $99.11 \%$ of accuracy,99.22\% of precision, $98.98 \%$ of recall and $99.09 \%$ of F1 score.

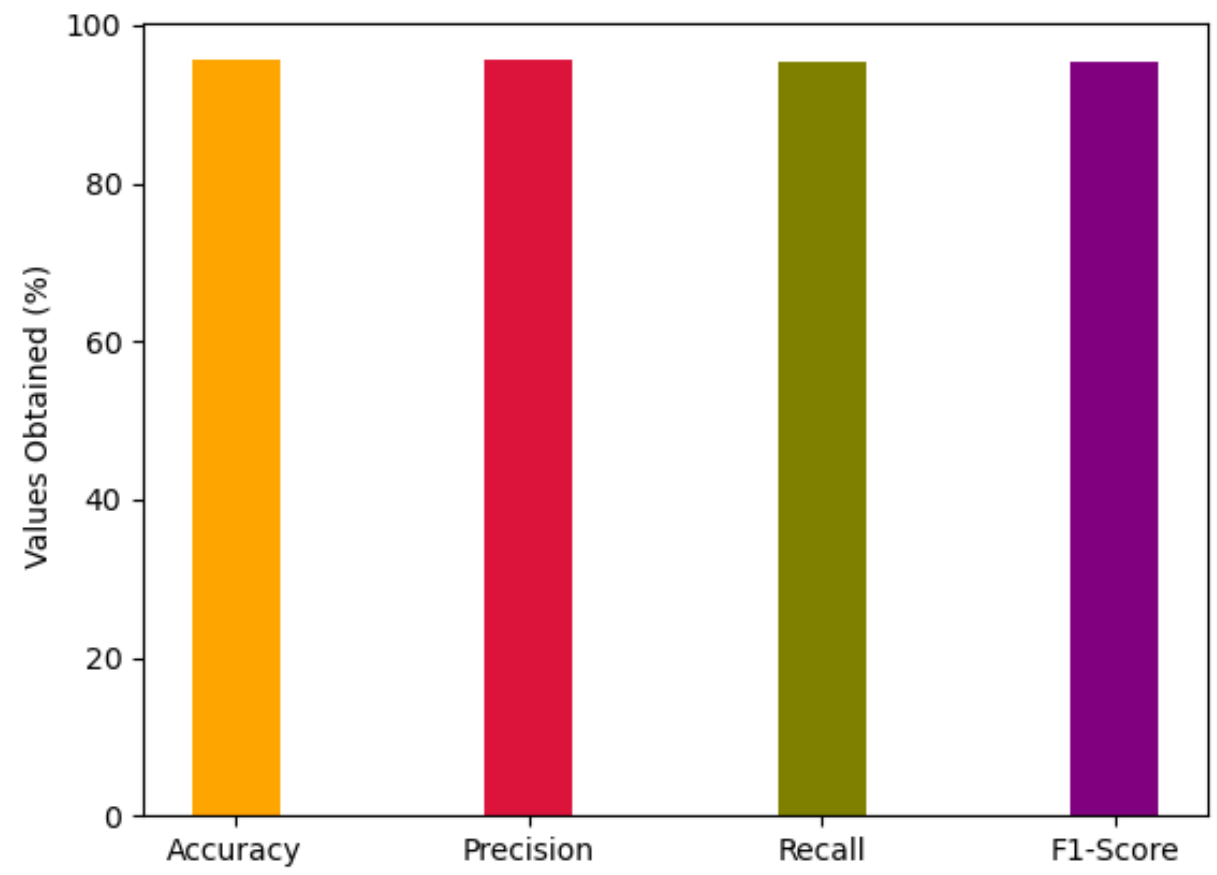

Figure 13: SURF feature usingadaboost classifier

The figure 13 shows the SURF feature usingadaboost classifier whereas, $\mathrm{X}$ axis shows the parameters to be used for analysis and $\mathrm{Y}$ axis shows the values obtained in percentage. The classifier achieves $95.54 \%$ of accuracy, $95.57 \%$ of precision, $95.35 \%$ of recall and $95.45 \%$ of F1 score 


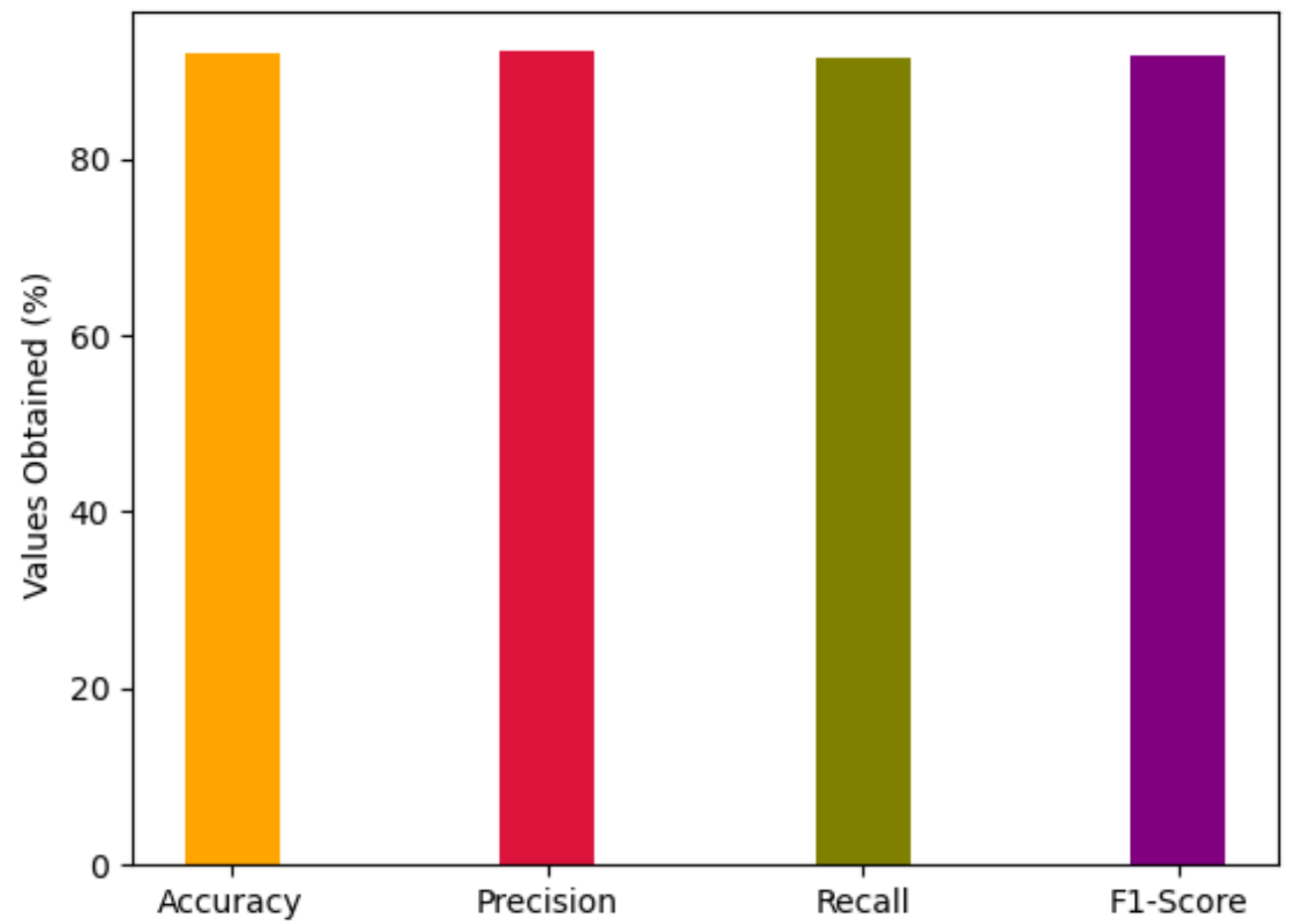

Figure 14: SURF feature usinggradient boosting classifier

The figure 14 shows the SURF feature usinggradient boosting classifier whereas, $\mathrm{X}$ axis shows the parameters to be used for analysis and Y axis shows the values obtained in percentage. The classifier achieves $91.96 \%$ of accuracy, $92.19 \%$ of precision, $91.5 \%$ of recall and $91.77 \%$ of $\mathrm{F} 1$ score.

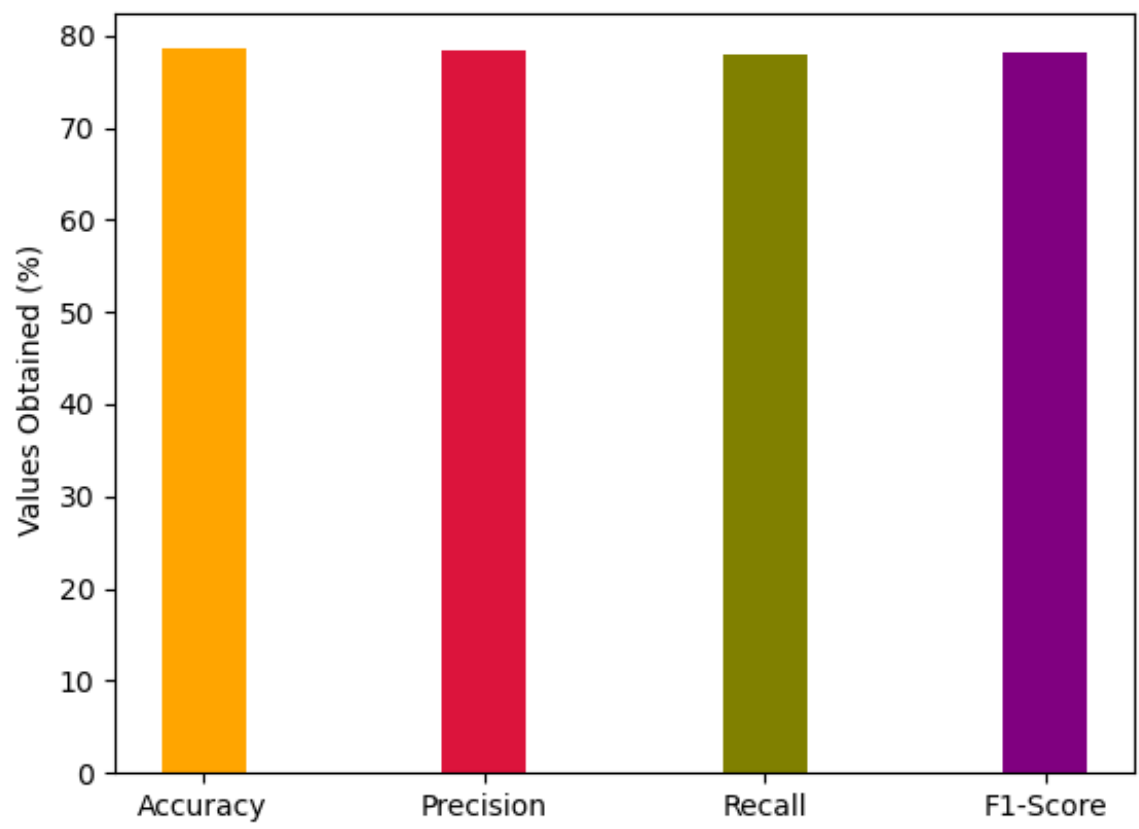

Figure 15: SURF feature usingMLP classifier

The figure 15 shows the SURF feature usingMLP classifier whereas, $X$ axis shows the parameters to be used for analysis and $\mathrm{Y}$ axis shows the values obtained in percentage. The classifier achieves $78.57 \%$ of accuracy,78.3\% of precision, $78 \%$ of recall and $78.12 \%$ of $\mathrm{F} 1$ score. 
S.No

1. Benign

2.

4.

5.

3.

\section{Malignant}

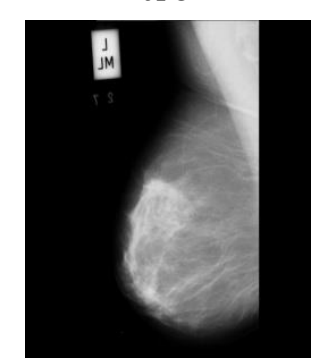

mdb095.jpg

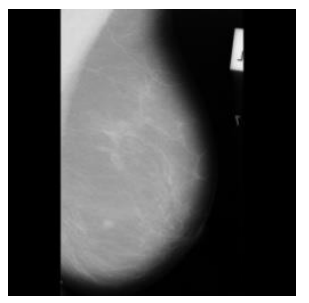

mdb256.jpg

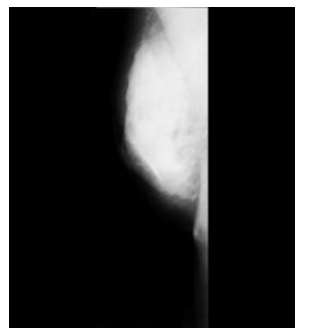

mdb179.jpg

Pre-Processed

Image
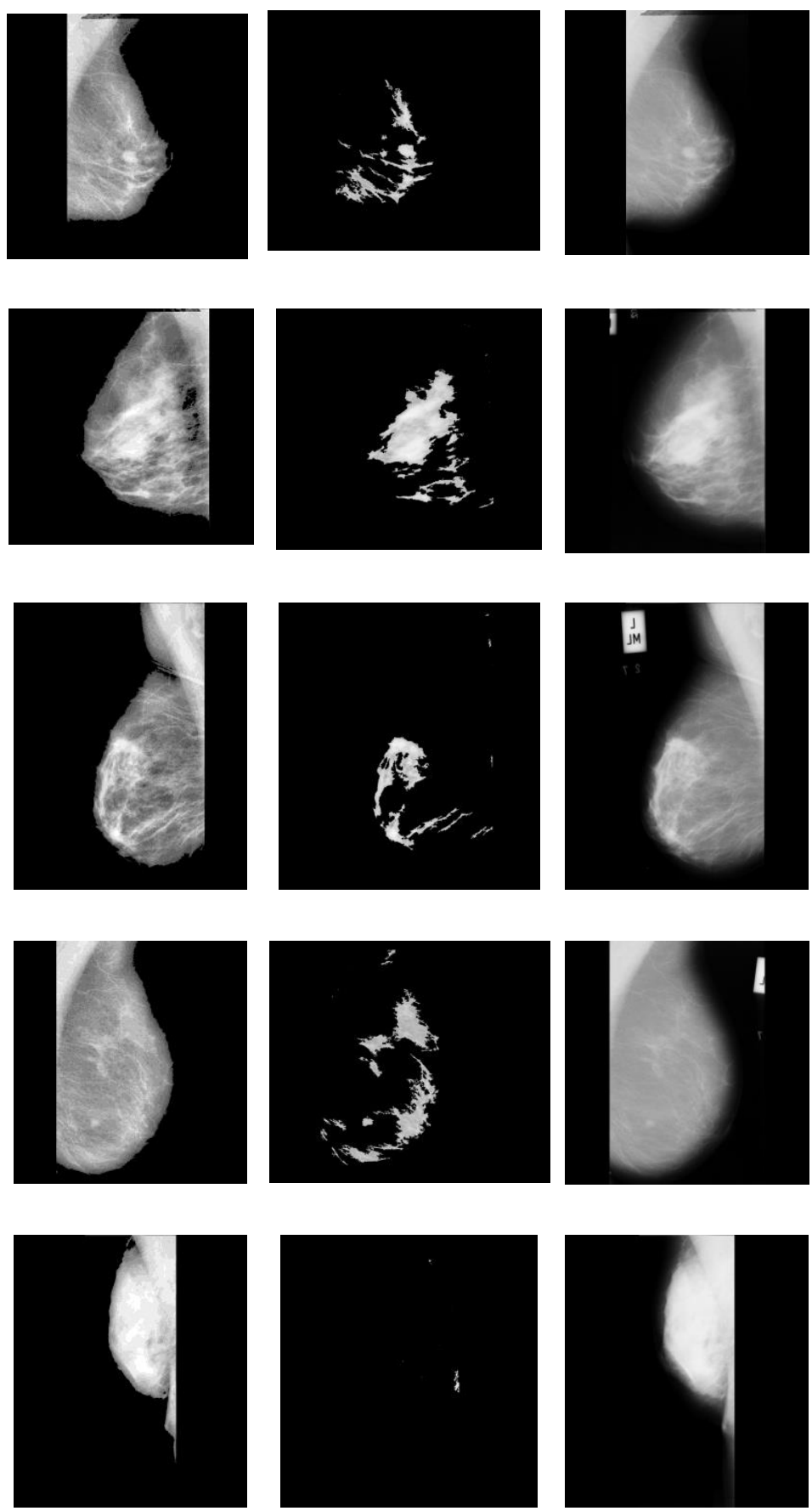

Figure 16: Output of various breast images

Figure 16 shows the output of various breast images in preprocessing, segmentation and feature extraction stage, whereas the input considered are benign and malignant images 


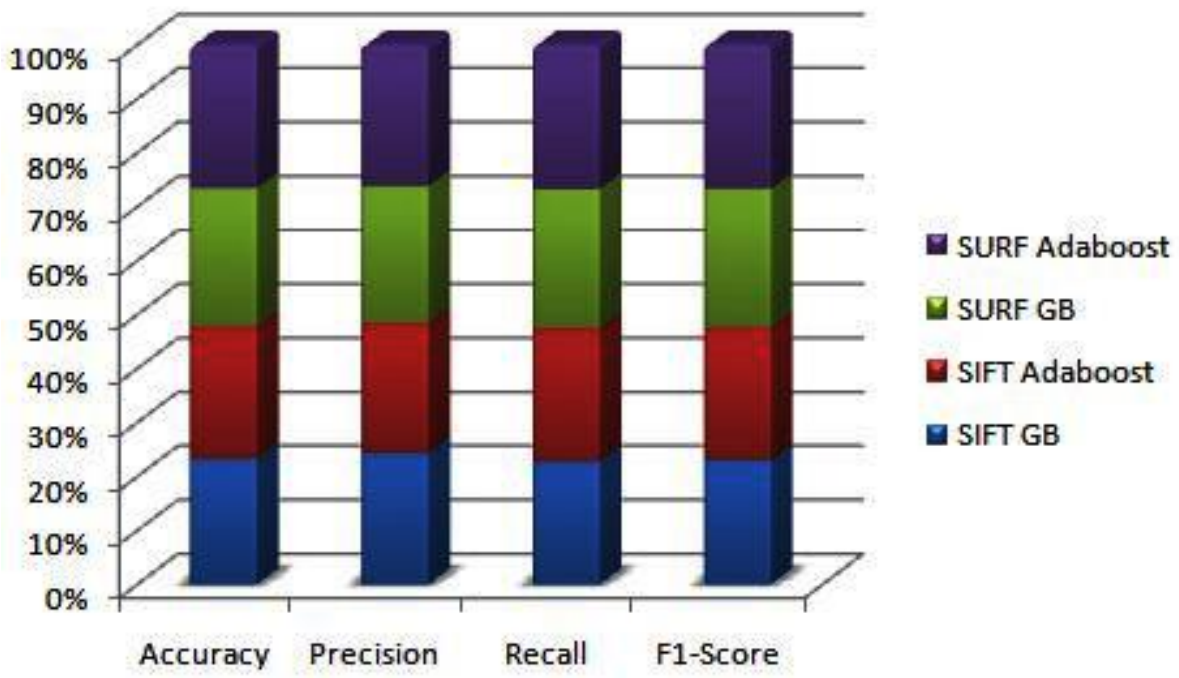

Figure 17: Overall comparison of SURF and SIFT feature classifier.

The figure 17 shows the overall comparison of SURF and SIFT feature in gradient boost and adaboost

\section{CONCLUSION}

Under medical application, finding the breast cancer by classification is most significant. The purpose of this research is to expand accurate organization structure to sort the regularity and irregularity of breast cancer with medical dataset. In this situation, feature extraction oriented classification is used to fragment the cancer and non cancer section. The obtained characteristic values are classified by using the adaboost and gradient classifier. The proposed method bring an effectual presentation by way of accuracy, precision, recall and F1-score.In the future work, the morphologhical study has to be done for promoting the overall classification rate

\section{REFERENCES}

4. Cruz-Roa A, Gilmore H, Basavanhally A, Feldman M, Ganesan S, Shih N. N andMadabhushi A, “Accurate and reproducible invasive breast cancer detection in whole-slide images: A Deep Learning approach for quantifying tumor extent",Scientific reports, vol.7, 2017.

5. Mambou S. J, Maresova P, Krejcar O, Selamat A and Kuca K,"Breast cancer detection using infrared thermal imaging and a deep learning model",Sensors, vol.18, no.9, 2018.

6. Yap M. H, Pons G, Martí J, Ganau S, Sentís M, Zwiggelaar R andMartí R, “Automated breast ultrasound lesions detection using convolutional neural networks",IEEE journal of biomedical and health informatics, vol.22, no.4, pp.1218-1226, 2017.

7. Lévy D and Jain A,"Breast mass classification from mammograms using deep convolutional neural networks",arXiv preprint arXiv:1612.00542, 2016.

8. Dai J, Li Y, He K and Sun J, “R-FCN: Object Detection via Region-based Fully Convolutional Networks", arXiv:1605.06409 [cs], 1605.06409, 2016.

9. Ren S, He K, Girshick R and Sun J,"Faster R-CNN: Towards real-time object detection with region proposal networks",In Advances in Neural Information Processing Systems, pp.91-99, 2015.

10. Lévy D and Jain A,"Breast Mass Classifcation from Mammograms using Deep Convolutional Neural Networks",arXiv preprint arXiv:1612.00542, 2016.

11. Rodríguez-Ruiz A, Krupinski E, Mordang J. J, Schilling K, Heywang-Köbrunner S. H, Sechopoulos I and Mann R. M,"Detection of breast cancer with mammography: effect of an artificial intelligence support system”, Radiology, vol.290, no.2, pp.305-314, 2019.

12. Sebai, Meriem, Tianjiang Wang and Saad Ali Al-Fadhli, "PartMitosis: A Partially Supervised Deep Learning Framework for Mitosis Detection in Breast Cancer Histopathology Images", IEEE Access, vol.8, pp.4513345147, 2020.

13. Zheng, Jing, Denan Lin, ZhongjunGao, Shuang Wang, Mingjie He and Jipeng Fan, "Deep Learning assisted Efficient AdaBoost Algorithm for Breast Cancer Detection and Early Diagnosis", IEEE Access, 2020. 
14. Suresh R, Rao A. N and Reddy B. E, "Detection and classification of normal and abnormal patterns in mammograms using deep neural network", Concurrency and Computation: Practice and Experience, vol.31, no.14, 2019.

15. Zhou, Juan, Lu- Yang Luo, Qi Dou, Hao Chen, Cheng Chen, Gong- Jie Li, Ze- Fei Jiang and Pheng- Ann Heng, "Weakly supervised 3D deep learning for breast cancer classification and localization of the lesions in MR images", Journal of Magnetic Resonance Imaging, vol.50, no.4, pp.1144-1151, 2019.

16. Saha M and Chakraborty C,"Her2Net: A deep framework for semantic segmentation and classification of cell membranes and nuclei in breast cancer evaluation. IEEE Transactions on Image Processing, vol.27, no.5, pp.2189-2200, 2018.

17. Carneiro, Gustavo, Jacinto Nascimento and Andrew P. Bradley,"Automated analysis of unregistered multiview mammograms with deep learning”, IEEE transactions on medical imaging, vol.36, no.11, pp.23552365, 2017.

18. Yap M. H, Pons G, Martí J, Ganau S, Sentís M, Zwiggelaar R andMartí R, “Automated breast ultrasound lesions detection using convolutional neural networks", IEEE journal of biomedical and health informatics, vol.22, no.4, pp.1218-1226, 2016. 